\title{
A Space-Time Semi-Blind Equalizer Based on Constant Modulus and Decision-Direction
}

\author{
Xiangyang Zhuang and A. Lee Swindlehurst \\ Department of Electrical and Computer Engineering \\ Brigham Young University, Provo, UT 84602 \\ cmail: $\{$ jeffz, swindle\}@ee.byu.edu
}

\begin{abstract}
This paper considers the problem of directly computing inverse filters that can equalize and separate each user in a multi-user multipath environment. Some known but very small number of training symbols, while alone unable to yield a satisfactory filter, provide reasonable initializations that not only may speed up the convergence of an adaptive blind algorithm, but also guide the algorithm to un easier recovery of weak users. Training for each user does not have to be simultaneous. Decision-direction mode can be used to refine the filter coefficients after the phase ambiguity inherent to a blind approach is corrected for. Simulation results are provided to demonstrate the method's effectiveness.
\end{abstract}

\section{INTRODUCTION}

In order to detect ISI-affected sequences from multiple co-channel users who occupy the same time slot and frequency band without spectrum spreading. we can estimate all channels followed by either a joint maximum likelihood sequence detection or a channel inversion to obtain filters for each user. Alternatively. we may directly compute the filters or estimate the sequences. Conventionally, the acquisition of channels or filters is accomplished using training data. Training sequence lengths are kept as short as possible to avoid significant reduction of the throughput efficiency. However, a short training sequence has a limited ability to "arerage out" the effects of noise and interference. Moreover, a simultaneous (cooperative) training interval is required for all co-channel users to estimate all channcls. This synchronized training scheme, even if possible. is undesirable since training intervals for all users have to be aligned and known symbols out of that interval can not be utilized. On the other hand, blind methods which can completely aroid the use of training often require more data. The knowledge of some known symbols from dedicated training or perhaps from synchronization overhead (or even payload symbols) can be used to improve the performance of blind methods. Training can be combined with the blind subspace criteria [4] or the blind maximum likelihood principle $[1,2,3]$, or can be exploited in the parameterization of a Markor sequence [6]. In [8] the whole sequence is treated as the superposition of known and unknown subsequences and row space of the data is used to directly estimate the symbols. Methods that directly compute an equalizing filter include one that combines training with the constant modulus cost function [5] and one that combines training with a second order subspace constraint [9]. So far, the above are all block methods and some involve iterations orer the whole block.

In this paper, a semi-blind sequential algorithm is proposed in which an adaptive blind method acquires the filters quickly from initializations set by a short training sequence. Decision-direction (DD) is then used to further refine the filter. We seek inverse filters because of the high complexity involved in the simultaneous channel estimation and the subsequent joint sequence detection for all users. Training from different users is allowed to occur at different time intervals. The adaptive algorithm [12] is derived from the $\mathrm{CM}(2,2)$ cost function and can be used for fast blind acquisition thanks to its surprising capability to converge within a very small amount of data. Even less data is required if the training provides a "good" initialization. In a near-far situation, a weak user is easier to recover if its training data is employed. Decision-direction is often very helpful too: if we start with the filter derived from a short training sequence and iterate over the data block several times using the method in [7], we may get the desired convergence. But convergence problems can occasionally occur depending on the initialization. In sequential processing. wrong decisions can lead to "catastrophic" divergence. If this occurs, we have to re-acquire the filter using training or preferably blindly as proposed here.

\section{DATA MODEL}

We first consider a single user transmitting through multiple channels resulting from either oversampling or using an antenna array. In the following model, $M$ denotes the number of sensors, $P$ the oversampling fac- 
tor, and $\mathbf{x}_{M P}(n)$ is a vector containing samples from all $M P$ sub-channels at time instant $n . L$ is the effective channel length in symbol periods. After cach sub-channel, an $E$-tap temporal filter is implemented. Hence the baseband sampled signal present at all $M P E$ taps of the space-time filter can be written as

$$
\begin{aligned}
\mathbf{x}(n) & =\left[\mathbf{x}_{M P}^{T}(n) \cdots \mathbf{x}_{. H P}^{T}(n-E+1)\right]^{T} \\
& =\left[\begin{array}{cccccc}
\mathbf{h}_{0} & \cdots & \mathbf{h}_{L-1} & \mathbf{0} & \mathbf{0} & \mathbf{0} \\
\mathbf{0} & \mathbf{h}_{0} & \cdots & \mathbf{h}_{L-1} & \mathbf{0} & \mathbf{0} \\
\vdots & \ddots & \ddots & \ddots & \ddots & \vdots \\
\mathbf{0} & \mathbf{0} & \mathbf{0} & \mathbf{h}_{0} & \cdots & \mathbf{h}_{L-1}
\end{array}\right] \mathbf{s}(n)+\mathbf{n}(n) \\
& =\mathcal{H}_{1} \mathbf{s}(n)+\mathbf{n}(n)
\end{aligned}
$$

where the $M P$-tuple vector $\mathbf{h}_{i}$ contains the channel coefficients at the delay index $i, \mathbf{s}(n)=[s(n) \cdots s(n-E-$ $L+2)]^{T}$ is the signal vector consisting of the sequence at different time shifts and $\mathbf{n}(n)$ is the noise vector $\mathbf{n}(n)=\left[\mathbf{n}_{M P}^{T}(n) \cdots \mathbf{n}_{M P}^{T}(n-E+1)\right]^{T}$.

For the case of $d$ co-channel users, we have

$$
\mathbf{x}(n) \equiv\left[\mathcal{H}_{1} \cdots \mathcal{H}_{d}\right]\left[\begin{array}{c}
\mathbf{s}_{1}(n) \\
\vdots \\
\mathbf{s}_{d}(n)
\end{array}\right]+\mathbf{n}(n)=\mathcal{H} \mathbf{s}+\mathbf{n}(n)
$$

where $\mathcal{H}$ is a complex matrix of dimension $M P E \times$ $\left(d E-d+\sum_{i=1}^{d} L_{i}\right)$. Without loss of generality; we take the power of each user's signal to be unity since the actual signal power can always be absorbed into the channel. Therefore, the coefficients of $\mathcal{H}$ corresponding to strong users are numerically larger than those of weak uscrs. The channel matrix $\mathcal{H}$ is assumed to have more rows than columns, which can always be attained through the choice of a large enough $E$. The cases of "wide" $\mathcal{H}$ (e.g., single-channel) and ill-conditioned $\mathcal{H}$ are discussed in [10]. The blind algorithm further assumes the user sequences are self- and mutually independent.

\section{BLIND EQUALIZATION METHOD}

In the Fixed-Window Constant Modulus Algorithm (FWCMA-2) of [11], equalizing filters are computed through iterations based on a fixed-length block of data. Each iteration consists of the following two steps:

$$
\begin{aligned}
& \mathbf{w}_{k+1}=3 \mathbf{w}_{k}-E^{\dagger}\left(\mathbf{x} \mathbf{x}^{H}\right) E\left(\mathbf{x x}^{H}\left|\mathbf{w}_{k}^{H} \mathbf{x}\right|^{2}\right) \mathbf{w}_{k} \\
& \mathbf{w}_{k+1}=\mathbf{w}_{k+1} / \sqrt{\mathbf{w}_{k+1}^{H} E\left(\mathbf{x x}^{H}\right) \mathbf{w}_{k+1}}
\end{aligned}
$$

where $\mathbf{w}_{k}$ denotes the filter at iteration- $k$ and $\beta=2,3$ for complex circular and real-valued modulation respectively. The symbol $(\cdot)^{\dagger}$ denotes the pseudo-inverse operation which is required theoretically in the absence of noise. In implementation, a plain inversion with suitable diagonal loading is used which amounts to adding some Gaussian noise. The first step is derived from the popular $\mathrm{CM}(2,2)$ (Godard) cost function and the second one serves as a post-normalization $\left(\|\mathbf{g}\|_{2}^{2}=1\right)$ of the combined equalizer/channel global response $\mathbf{g}^{H} \triangleq \mathbf{w}^{H} \mathcal{H}$.

When $\mathcal{H}$ is of full column rank and the input sequence is i.i.d., the post- and pre-iteration ratio between any two elements of $g$ was shown in [11] to be

$$
\frac{g_{(k+1) i}}{g_{(k+1) j}}=\frac{g_{k i}\left|g_{k i}\right|^{2}}{g_{k j}\left|g_{k j}\right|^{2}}
$$

from which we can see that the convergence is monotonic; i.e., the largest coefficient of the initialization $g_{0}$ converges to magnitude 1 (after normalization) and the rest go to zero monotonically while their relative numerical order is preserved. Since the post-iteration ratio is the cube of the previous value, the convergence is super-fast and only a few iterations are usually necessary. We can see that the algorithm always tries to reach the global minimum that is closest (in Euclidean distance) to $\mathbf{g}_{0}$, avoiding the attraction of other minima which are present due to the multi-modal cost function. Furthcrmore, the final delay at which the sequence will be recovered is determined by the peak position of $\mathbf{g}_{0}$, which is determined by the filter initialization $\mathbf{w}_{0}$. As we have shown in [11], the output SNR associated with different delays may be dramatically different due to noise amplification which is determined by the eigenstructure of the channel matrix $\mathcal{H}$. Consequently: multiple filters at different delays may be desired. Additionally, in a multi-user environment, we must obtain filters that recover all users. In these cases, a reliable relationship between initialization and the expected final delays is so critical that otherwise the same delays or only strong users will be recovered even when different initializations are used.

When sequential processing is desired, existing variations of CMA all resort to some approximation such as replacing the expectation with its instantaneous estimate. As a result, unpredictable and slow convergence behavior is often observed. In [12], an adaptive implementation of FWCMA-2 is proposed which preserves the monotonic characteristic. In that algorithm, we notice that $\left[E\left(\mathbf{x x}^{H}\right)\right]^{-1}$ in (3) can be sequentially updated as in the RLS algorithm and the only computation in (3) requiring the stored data block is the term $E\left(\mathbf{x x}^{H}\left|\mathbf{w}_{k}^{H} \mathbf{x}\right|^{2}\right)$. which is re-computed at each iteration based on the previous filter result $\mathbf{w}_{k}$. Using the Kronecker product, we have the useful transformations:

$$
\begin{aligned}
\mathbf{x} \mathbf{x}^{H} & =\operatorname{unvec}\left(\mathbf{x} \otimes \mathbf{x}^{*}\right) \\
\left|\mathbf{w}^{H} \mathbf{x}\right|^{2} & =\left(\mathbf{x} \otimes \mathbf{x}^{*}\right)^{H}\left(\mathbf{w} \otimes \mathbf{w}^{*}\right)
\end{aligned}
$$

where "unvec" is the inverse operation of row stacking, " $\otimes$ " denotes the Kronecker product and "*" denotes 
conjugation. Thus,

$$
E\left(\mathbf{x x}^{H}\left|\mathbf{w}_{k}^{H} \mathbf{x}\right|^{2}\right)=\operatorname{unvec}\{\underbrace{E\left[\left(\mathbf{x} \otimes \mathbf{x}^{*}\right)\left(\mathbf{x} \otimes \mathbf{x}^{*}\right)^{H}\right]}_{\triangleq c} \cdot\left(\mathbf{w}_{k} \otimes \mathbf{w}_{k}^{*}\right)\}
$$

The fourth order moment matrix $G$ defined above can be adaptively accumulated, which allows us to free the iteration from the actual data. We have the flexibility to choose when we want to iterate, from which initializations, how many times we want to iterate and how many filters are computed in parallel from different initializations. The solution can be reached through a few iterations as soon as the elapsed sequence is sufficiently i.i.d. . The great flexibility comes at a price of computational complexity. The update of $G$ is of complexity $\mathcal{O}\left((M P E)^{4}\right)$ flops per symbol. Recognizing the multiple Hermitian symmetry of $G$, all complex flops can be reduced to real flops.

\section{SEMI-BLIND EQUALIZATION}

If we seek a direct semi-blind equalizer or channel estimate, a least-squares (LS) criterion is most often used for the known part of the data. This LS criterion can be used as an auxiliary constraint for the blind cost function, or vice versa. The method of Lagrange multipliers will transform a constrained optimization problem to an unconstrained composite criterion.

A straightforward cost function that combines the LS criterion with the $\mathrm{CM}(1,2)$ cost function is proposed in $[5]$ as follows:

$J_{\mathbf{w}}=\frac{1}{N_{k}} \sum_{i=n_{0}}^{n_{0}+N_{k}-1}\left|\mathbf{w}^{H} \mathbf{x}_{i+D}-s(i)\right|^{2}+\frac{\rho}{N} \sum_{i=1}^{N}\left(\left|\mathbf{w}^{H} \mathbf{x}_{\mathbf{i}}\right|-1\right)^{2}$

where $\rho$ is some regularization coefficient, $n_{0}$ is the starting position of the known symbols and $N_{k}\left(N_{u}\right)$ is the number of known (unknown) symbols. An important parameter is $D$, the delay (relative to the current time instant) at which the sequence will be recovered. It ranges from 0 to $\left(d E-d+\sum_{i=1}^{d} L_{i}\right)$ and is specified in advance. In multi-user cases, $D$ also indicates the user index (refer to model (2)). In the cost function above, $\operatorname{CM}(1,2)$ is chosen over the $\operatorname{CN}(2,2)$ cost function because the latter enforces the CM constraint more strictly and may undesirably dominate the composite criterion. But like $\mathrm{CM}(2,2), \mathrm{CM}(1,2)$ is also a multi-modal cost function and has multiple minima in the vicinity of the Wiener solutions associated with different delays. The $w$ that minimizes the LS term of the composite criterion will recover the sequence at delay $D$, but it may not be the one that globally minimizes the $\mathrm{CM}(1,2)$ term since other delays may offer a smaller cost than delay $D$. The conflict incurred by simply adding the two terms has to be dealt with by choosing $\rho$ properly. We can imagine that the choice of $\rho$ will depend on many factors such as the SNR, $D, N_{k}, N$, etc.. A simulation-based characterization of the $\rho$ factor is reported in [5] for the particular parameters therein. Due to the non-linearity of the cost function, a closedform solution is difficult to obtain. A Gauss-Newton type of off-line algorithm based on the gradient must be used. The search process can take many iterations and may be trapped in an undesired minimum.

Noticing the drawback of using a multi-modal constraint, the authors of [9] replace the $\mathrm{CM}(1,2)$ part by a constraint that can be minimized only by the filter with delay $D$ (i.c., the only null vector of some second order statistics matrix). Instead of totally abandoning the $\mathrm{CM}(1,2)$ term as they did, our strategy is to simply apply the block FWC.MA-2 with the initialization obtained from training. Thanks to the built-in delay control mechanism of FWCMA-2, if the initialization puts the starting $g_{0}$ in the basin of delay $D$, the blockiterative algorithm will converge to that expected minimum quickly. The initialization is still obtained using the LS criterion as in [5]. A regularization must be used for very short training sequences (in particular when $N_{k}<M P E$ ), hence the initial filter estimate is

$$
\mathbf{w}=\left(R_{t r}+\delta I\right)^{-1} \mathbf{p}_{t r}
$$

where $\delta$ is a small number (e.g., $0.01 \sigma_{\text {noise }}^{2}$ ), and

$$
\begin{aligned}
& R_{t r}=\sum_{i=n_{0}}^{n_{0}+y_{k}-1} \mathbf{x}_{i+D} \mathbf{x}_{i+D}^{H} \\
& \mathbf{p}_{t r}=\sum_{i=n_{0}}^{n_{0}+x_{k}-1} s_{i}^{*} \mathbf{x}_{i+D}
\end{aligned}
$$

Of course, the LS solution depends on the SNR, $D$ and $N_{k}$ and it is often not satisfactory as a final solution especially when $N_{k}<M P E$, but it may lie in the basin of the expected delay $D$. If $N_{k}$ is too short, $\mathbf{s}_{k}$ may be just a trivial transformation of a shifted version of itself or of a partial sequence of another user. Then, the LS solution may not serve as a good initialization. After FWCMA-2 converges to a filter $w_{c}$ : a decision-directed iterative procedure such as in $[\bar{i}]$ may be used; i.e., based on $\mathbf{w}_{c}$, tentative symbol decisions are made which are used in turn to compute a new Wiener filter. The procedure is repeated until no change in the decisions are observed. This procedure will improve the BER performance, especially in low SNR situations. Before $\mathbf{w}_{c}$ is used, we can coarsely (due to the noise) compensate for the phase rotation factor inherent to blind methods, thanks to the known symbols. The rotation ambiguity can be easily calculated using $\min _{a}\left|\alpha^{*} \mathbf{w}_{c}^{H} X_{t r}-s_{t r}\right|$, whose solution is $\alpha=\mathbf{w}_{c}^{H} \cdot \mathbf{p}_{t r} /\left|\mathbf{w}_{c}^{H} R_{t r} \mathbf{w}_{c}\right|^{2}$.

A blind approach must be used when the LS solution from training alone is not satisfactory: On the 
other hand, the benefit of training to the blind approach is manifold. First, the rotation can be approximately corrected to make symbol decisions. Second, the initialization set by training makes it easier to recover a weak user in a near-far situation. Since the user power is absorbed into the $\mathcal{H}$ and a simple spike initialization corresponds to a $\mathrm{g}_{0}$ which is some row of $\mathcal{H}$, the final delay which is determined by the peak position of $g_{0}$ must belong to a strong user at a certain delay. Weak users can only be recovered through a deflation approach [11]. However, some training symbols from a weak user can set the initial point in the basin corresponding to that user and the built-in delay control will lead to the desired recovery. Third, the initialization also has a significant impact on the number of iterations required. We can see from (5) that the algorithm will enter the so-called saddle point when two or more elements of $\mathbf{g}_{0}$ share the largest value. Although exactly equal coefficients are rare, nearly equal peaks will make the algorithm converge much more slowly. This could happen when users are received with similar powers and a spike initialization is used. Finally, a good initialization can reduce the number of symbols required to achieve convergence and increase the reliability of converging to the expected user/delay. For example, if the initialization can alieady yield a constant modulus output (i.e., $\left|\mathbf{w}_{k}^{H} \mathbf{x}\right|^{2}=1$ ), we can see from (3) that the iteration will result in no change even though the sequence is still too short to establish the i.i.d. property: Other factors that also influence the number of symbols required are SNR and the accuracy of the i.i.d. assumption for the particular realization of the user sequence.

Our adaptive semi-blind approach basically uses the adaptive FWCMA-2 for fast filter acquisition and relies on decision-direction (DD) for fine tuning. An outline of the algorithm is given below:

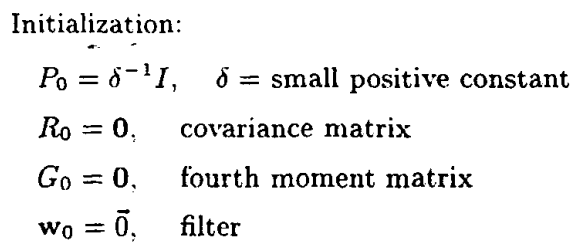

At each instant before convergence $n=1,2, \cdots$, compute

$$
R_{n}=\lambda R_{n-1}+\mathbf{x}_{n} \mathbf{x}_{n}^{H}
$$

( $\lambda$ : forgetting factor, set $\lambda=1$ before convergence)

$G_{n}=\lambda G_{n-1}+\left(\mathbf{x}_{n} \otimes \mathbf{x}_{n}^{*}\right)\left(\mathbf{x}_{n} \otimes \mathbf{x}_{n}^{*}\right)^{H}$

$\mathbf{k}_{n}=\frac{\lambda^{-1} P_{n-1} x_{n}}{1+\lambda^{-1} \mathbf{x}_{n}^{H} P_{n-1} x_{n}}$

$P_{n}=\lambda^{-1} P_{n-1}-\lambda^{-1} \mathbf{k}_{n} \mathbf{x}_{n}^{H} P_{n-1}$

Training Phase: (assuming training occurs at the beginning of a burst)

$$
\begin{aligned}
& \mathbf{w}_{n}=\mathbf{w}_{n-1}+\mathbf{k}_{n}\left(s_{n}-\mathbf{w}_{n-1}^{H} \mathbf{x}_{n+d}\right)^{*}, \text { for } n<=N_{k} \\
& \text { Check Convergence at Selected Check Points " } t ": \\
& \hline \text { Iterate using initialization } \mathbf{w}_{N_{k}} \text { as follows: } \\
& \mathbf{w}_{t, l+1}=3 \mathbf{w}_{t, l}-P_{t} \cdot \text { unvec }\left\{G_{t} \cdot\left(\mathbf{w}_{t, l} \& \mathbf{w}_{t, l}^{*}\right)\right\} \mathbf{w}_{t, l} \\
& \mathbf{w}_{t, l+1}=\mathbf{w}_{t, l+1} / \sqrt{\mathbf{w}_{t, l+1}^{H}\left(R_{t} / t\right) \mathbf{w}_{t, l+1}} \\
& \text { If "convergence can be reached" (discussed later) } \\
& \text { Correct rotation factor and switch to DD mode } \\
& \text { Else } \\
& \text { Try at next check point from initialization } \mathbf{w}_{v_{k}} \\
& \text { End }
\end{aligned}
$$

The matrix $G$, which must be updated at each time instant before convergence, is no longer needed in the DD mode where we can apply algorithms such as LMS, NLMS and RLS. Before convergence, the forgetting factor $\lambda$ is set to 1 for fastest acquisition, but the step-size $K$ decreases at a rate of $1 / n$. Thus when $\lambda=1$, the correct decisions made later will have an increasingly negligible influence. We want to set $\lambda<1$ if the RLS algorithm is used in the DD mode.

Since we do not have to do iterations at all time instants, we can iterate only at selected points for computational savings. At those check points, an implementation issue is how to decide if convergence is attained. Since we can not use the unobservable global response, we propose to measure the change in $\mathbf{g}$ betwecn two consecutive iterations, i.e.,

$$
\left\|\Delta \mathbf{g}_{k}\right\|^{2}=\left\|\mathbf{g}_{k}-\mathbf{g}_{k-1}\right\|^{2} \approx\left(\mathbf{w}_{k}-\mathbf{w}_{k-1}\right)^{H} R_{x}\left(\mathbf{w}_{k}-\mathbf{w}_{k-1}\right)
$$

If after an allowed maximum number of iterations (say $q),\left\|\Delta \mathbf{g}_{q}\right\|^{2}$ is still larger than a pre-specified threshold $\eta$, a failure is declared for the attempt at this sample. Because of the super-fast convergence rate, a very small $\|\Delta \mathbf{g}\|^{2}$ can be reached in just a few iterations from initializations that are not close to saddle points. Also, since $\|\Delta \mathbf{g}\|^{2}$ should decrease monotonically, whenever an increase in $\|\Delta \mathbf{g}\|^{2}$ is observed after an iteration, a failure can be declared with no need for further iteration. We find this implementation strategy effective and reliable, though other methods are possible, such as investigating $\|\Delta \mathbf{w}\|^{2}$, or examining the MSE performance of the tentative decisions based on that $\mathbf{w}$.

\section{SIMULATIONS}

The case of two simultaneous users in a "Bad Urban" environment is simulated. Each user has eight rays arriving in two clusters with the total signal power of the first cluster set to be twice as high as that of the second cluster. The modulation is QPSK with a raised cosine pulse shape. After truncation, the channel lengths are $6 \mathrm{~T}$ and $5 \mathrm{~T}$, respectively. The space-time receiver consists of an array of four $(M=4)$ half-wavelengthspaced antennas each with $\mathrm{T} / 2$ oversampling $(P=2)$, 
and a two-tap temporal transversal filter after each of the $M P=8$ sub-channels. Hence the channel matrix $\mathcal{H}$ is $16-$ by-13. An extremely difficult near-far situation is simulated with user-1 $20 \mathrm{~dB}$ stronger than user2. which results a large condition number (292) for $\mathcal{H}$. The received SNR of user-2, defined as $S N R=$ $10 \log _{10}\left(\left\|\mathcal{H}_{2}\right\|_{F}^{2} /\left(\sigma_{n}^{2} M P E\right)\right)$, is set to $20 \mathrm{~dB}$ though its SINR is less than $-20 \mathrm{~dB}$. Our performance measure is the residual ISI defined as $I S I=\frac{\sum_{i}\left|g_{i}\right|^{2}-\max _{i}\left(\left|g_{i}\right|^{2}\right)}{\max _{i}\left(\left|g_{i}\right|^{2}\right)}$. When we recover user-1 with some known symbols, no knowledge of user-2 is required (i.e., treated as a blind interferer), and vice versa. We simply designate the first $N_{k}=12$ (<MPE) random symbols at the beginning of a burst to be known. During our blind acquisition, the maximum iteration number is $q=8$ and $\eta=0.001$. Our check points are every $n$ for $n>30$ and $\lambda$ is set to 0.98 in the tracking mode.

A typical trial is shown in Figure 1. Also included is the performance for the RLS algorithm fed with tentative decisions at every $n>N_{k}$, RLS with ideal decision feedback, and the Wiener bound obtained assuming an entire block of 300 known symbols. For the stronger user (upper.subplot), 12 known symbols can already result in a "good" filter whose residual ISI is shown as the flat line till the point at which convergence is declared. All of the tentative decisions after $N_{k}$ in this case are correct and can be used as if nore training were provided. For the weak user (lower subplot), however, unreliable tentative decisions result in very slow convergence if not divergence. Our semi-blind approach converges, though as expected. more data is required. Meanwhile, the blind algorithm can only recover a weak user after all stronger signals are successively recovered first [11]. Due to the different initializations set by the random known symbols in our 200 Monte Carlo trials, the number of symbols required to attain convergence is also different from trial to trial. In Figure 2, we plot the percentage of trials in which convergence is attained within a certain amount of data. Out of all the trials, we observed three cases where convergence was not attained for the weak user due to poor initialization set by the short training data. However, the RLS algorithm with tentative DD failed in more than $50 \%$ of the trials.

\section{REFERENCES}

[1] J. Ayadi, E. de Carralho, and D. Slock. Blind and semiblind maximum likelihood methods for FIR multichannel identification. Proc. IEEE ICASSP, 5:3185-3188, 1998.

[2] E. de Carvalho and D. Slock. Cramer-rao bounds for semiblind, blind and training sequence based channel estimation. Proc. IEEE SPAWC Workshop, pages 129-132, 1997.

[3] E. de Carvalho and D. Slock. Semi-blind maximum likelihood blind multichannel estimation with Gaussian prior for the symbols using soft decisions. Proc. IEEE VTC, pages 1563-1567, 1998.
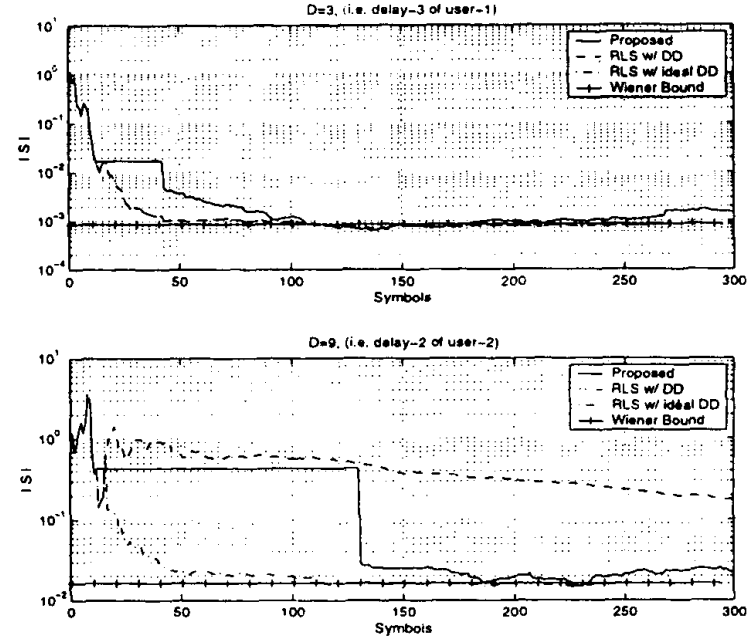

Figure 1: Acquisition and Tracking Behavior with 12 Known Symbols (User-1 is 20dB stronger than user-2)

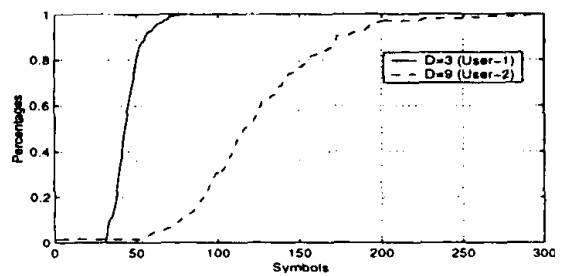

Figure 2: Probability that Convergence is Achieved within a Certain Number of Symbols

[4] A. Gorokhov and P. Loubaton. Semi-blind second order identification of convolutive channels. Proc. IEEE ICASSP. pages 3905-3908. 1997 .

[5] A. M. Kuzminskiy, L. Fety, P. Forster, and S. Mayrargue. Regularized semi-blind estimation of spatio-temporal filter coefficients for mobile radio communications. Proc. IEEE GRETSI, Grenoble. pages 127-130. September 1997.

[6] A. M. Kuzminskiy and D. Hatzinakos. Stochastic maximum likclihood methods for semi-blind channel estimation. IEEE Signal Process. Letter, pages 21-24, January 1998.

[7] A. L. Swindlehurst, S. Daas, and J. Yang. Analysis of a decision directed beamformer. IEEE Trans. Signal Process., 43:2920-2927. December 1995.

[8] A. L. Swindlehurst and J. Gunther. Direct semi-blind symbol estimation for multipath channels. Proc. 32nd A silomar Conferences on Signals: Systems, and Computers; 1998.

[9] A. Touzni, I. Fijalkow, and J. P. LeBlanc. Semi-blind spatiutemporal equalization of FIR filters with controlled delay: Proc. IEEE DSP Workshop, August 1998.

[10] X. Zhuang and A. L. Swindlehurst. Multi-user blind equalization via the fixed window constant modulus algorithm. In preparation.

[11] X. Zhuang and A. L. Swindiehurst. Fixed window constant modulus algorithms. Proc. IEEE ICASSP. March 1999.

[12] X. Zhuang and $A$. L. Swindlehurst. Fixed window constant modulus algorithms: Adaptive implementations. Proc. IEEE SPAWC Workshop, May 1999. 